bronchopulmonary dysplasia and restore lung function through macrophage immunomodulation. Am J Respir Crit Care Med 2018; 197:104-116.

2. Hansmann G, Fernandez-Gonzalez A, Aslam M, Vitali SH, Martin T, Mitsialis SA, et al. Mesenchymal stem cell-mediated reversal of bronchopulmonary dysplasia and associated pulmonary hypertension. Pulm Circ 2012;2:170-181.

3. Grisafi D, Pozzobon M, Dedja A, Vanzo V, Tomanin R, Porzionato A, et al. Human amniotic fluid stem cells protect rat lungs exposed to moderate hyperoxia. Pediatr Pulmonol 2013;48:1070-1080.

4. Lee C, Mitsialis SA, Aslam M, Vitali SH, Vergadi E, Konstantinou G, et al. Exosomes mediate the cytoprotective action of mesenchymal stromal cells on hypoxia-induced pulmonary hypertension. Circulation 2012 126:2601-2611.

5. Reiner AT, Witwer KW, van Balkom BWM, de Beer J, Brodie C, Corteling RL, et al. Concise review: developing best-practice models for the therapeutic use of extracellular vesicles. Stem Cells Transl Med 2017; 6:1730-1739.

6. Lane RE, Korbie D, Anderson W, Vaidyanathan R, Trau M. Analysis of exosome purification methods using a model liposome system and tunable-resistive pulse sensing. Sci Rep 2015;5:7639.

Copyright (C) 2018 by the American Thoracic Society

\section{Reply to Muraca et al.}

\section{From the Authors:}

The point raised by Muraca and colleagues regarding the purity of the extracellular vesicle preparations used in our recent studies (1) is very relevant to the exciting challenges we face as we translate research on mesenchymal stem/stromal cell (MSC) exosomes from the bench to the bedside. On the bench, one can assess the therapeutic effects of MSC exosomes using unpurified preparations. Indeed, many functional studies in preclinical models of disease employ crude extracellular vesicle fractions, such as the heterogeneous population obtained as pellets through differential ultracentrifugation or polyethylene glycol-induced extracellular vesicle sedimentation.

As we move forward to large-scale clinical trials, we should thoughtfully deliberate on the optimal purity of MSC exosome preparations, especially if such preparations are to be used in a neonatal ICU. Navigating the evolving regulatory landscape pertinent to this novel and promising therapeutic can be daunting, and although we recognize that U.S. and European Union agencies may differ in their requirements, the purity and consistency of the final pharmaceutical will probably be of paramount importance. Therefore, optimization considerations should factor in not only the cost but also the consistency, efficacy, and safety of the final pharmaceutical product. Safety considerations aside, it is clear that reproducibility in manufacturing processes and final product uniformity will make possible the design of simplified and expeditious potency assays and permit accurate dosage calculations.

In our experience, when functional activity is assessed in vivo, isolation of the exosomal fraction by density flotation results in more consistent potency metrics compared with other preparation methods (1). We clearly agree with Muraca and colleagues that this fractionation procedure does not lend itself to high-throughput

Originally Published in Press as DOI: 10.1164/rccm.201710-2066LE on November 21, 2017 applications, as, for example, size-exclusion chromatography traditionally does in industrial settings. Therefore, although we are using density floatation in our preclinical studies to ensure the consistency of our preparations, we do not expect the methodology to be used for large-scale production. What we do expect is that data from preclinical studies using fractionated extracellular vesicles will enable a comprehensive molecular and functional characterization of the active MSC exosome species and the factors that impact their biogenesis. This information will guide the industry in the design of manufacturing flowcharts, leading to the production of MSC exosome-based pharmaceuticals that will be optimized in terms of consistency, safety, efficacy, and cost. The specifics of such large-scale methodologies are, of course, beyond the scope of our present report.

We have discussed some of the above concepts in our recent reviews $(2,3)$.

Author disclosures are available with the text of this letter at www.atsjournals.org.

S. Alex Mitsialis, Ph.D.

Gareth R. Willis, Ph.D.

Angeles Fernandez-Gonzalez, Ph.D.

Stella Kourembanas, M.D.

Boston Children's Hospital

Boston, Massachusetts

and

Harvard Medical School

Boston, Massachusetts

\section{References}

1. Willis GR, Fernandez-Gonzalez A, Anastas J, Vitali SH, Liu X, Ericsson M, et al. Mesenchymal stromal cell exosomes ameliorate experimental bronchopulmonary dysplasia and restore lung function through macrophage immunomodulation. Am J Respir Crit Care Med 2018;197:104-116.

2. Willis GR, Kourembanas S, Mitsialis SA. Toward exosome-based therapeutics: isolation, heterogeneity, and fit-for-purpose potency. Front Cardiovasc Med 2017;4:63.

3. Willis GR, Kourembanas S, Mitsialis SA. Therapeutic applications of extracellular vesicles: perspectives from newborn medicine. Methods Mol Biol 2017;1660:409-432.

Copyright (C) 2018 by the American Thoracic Society

\section{Erratum: Effects of Age and Disease Severity on Systemic Corticosteroid Responses in Asthma}

The article by Phipatanakul and colleagues, published in the June 1 , 2017 , issue of the Journal (1), contained errors in the published spirometry results. The authors have informed us that derived spirometry parameters (percent of predicted) were incorrectly calculated. Derived parameters were calculated using the 2012 Global Lung Initiative (GLI) equations. However, because of a computer programming error, the GLI equations for Other/Mixed ethnicity were inadvertently applied to all white subjects instead of using the correct equations for white individuals. These errors produced small changes in the data dependent on the GLI equations but do not change the article's conclusions in any material way. 